\title{
Adenocarcinoma de vesícula biliar presentándose como síndrome de Trousseau y ganglio de Virchow-Troisie
}

\section{Gallbladder adenocarcinoma presenting as Trousseau syndrome and Virchow-Troisie's node}

Víctor A.Vargas-Rubio', Melissa N. Merizalde-Burneo², Edwin Hidalgo-Arroyo ${ }^{3}$, Cesar Chian-García ${ }^{4}$
Vargas-Rubio V, Merizalde-Burneo M, Hidalgo-Arroyo E, Chian-García C. Adenocarcinoma de vesícula biliar presentándose como síndrome de Trousseau y ganglio de Virchow-Troisie.. Rev Soc Peru Med Interna. 2020;33(3): 113-116.

https://doi.org/10.36393/spmi.v33i3.549

\begin{abstract}
RESUMEN
Mujer de 52 años que presentó dolor y aumento de volumen en el miembro inferior derecho, adenopatía supraclavicular izquierda y anemia microcítica e hipocrómica. Se descartó pérdidas gastrointestinales por endoscopias digestivas alta y baja normales. Se realizó una tomografía de abdomen que mostró irregularidad a nivel de la vesícula biliar, cuya biopsia resultó en un adenocarcinoma. Se concluye que se trató de una paciente con una neoplasia maligna de vesícula biliar que se presentó como síndrome de Trousseau y ganglio de Virchow-Troisier.
\end{abstract}

Palabras claves:Adenocarcinoma, vesícula biliar, trombosis venosa profunda, síndrome de Trousseau, ganglio de Virchow-Troisier.

\section{ABSTRACT}

A 52-year-old female patient who presented pain and swelling in the right lower limb, a left-sided supraclavicular lymph node and microcytic hypochromic anemia. It was ruled out gastrointestinal blood loss through normal upper and lower endoscopies. The abdominal tomography showed an irregular pattern in the gallbladder, whose biopsy resulted in adenocarcinoma. It is concluded that it was a patient with a malignant neoplasm of the gallbladder who presented as a Trousseaus's syndrome and a Virchow-Troisier's lymph node.

Keywords: adenocarcinoma, gallbladder, Trousseau's syndrome, Virchow-Troisier's lymph node.

\section{INTRODUCCIÓN}

El carcinoma de vesícula biliar es una enfermedad letal con pobre pronóstico, siendo la lesión maligna más común del

Médico Internista. Servicio de Emergencia, Hospital Nacional Arzobispo Loayza, Lima, Perú.

2 Interna de medicina. Hospital Nacional Arzobispo Loayza. Facultad de Medicina, Universidad Peruana Cayetano Heredia, Lima, Perú.

3 Médico internista. Departamento de Medicina. Facultad de Medicina, Universidad Peruana Cayetano Heredia. Hospital Nacional Arzobispo Loayza, Lima, Perú.

4 Médico patólogo. Servicio de Patología. Facultad de Medicina, Universidad Peruana Cayetano Heredia. Hospital Nacional Arzobispo Loayza, Lima, Perú. tracto biliar y la quinta más común de las neoplasias del tracto gastrointestinal. ${ }^{1}$ El tipo histológico más frecuente es el adenocarcinoma hasta en $90 \%$ de los casos, siendo los otros tipos el papilar, de células escamosas, carcinoma de células pequeñas y el indiferenciado. ${ }^{2}$

Las metástasis más frecuentes son a ganglios regionales y los sitios de extensión de la enfermedad son el hígado y peritoneo. También se ha descrito metástasis a distancia en pulmón y pleura. ${ }^{3}$ El carcinoma de vesícula es asintomático hasta estadíos avanzados de la enfermedad, se puede presentar con cólicos biliares o colecistitis aguda y crónica, cuando aparece ictericia generalmente el cáncer es irresecable; $y$, también, se ha descrito sangrado gastrointestinal y obstrucción intestinal alta como forma de presentación. ${ }^{4}$

La metástasis de cáncer de vesícula biliar a ganglios cervicales izquierdos y relacionada a trombosis venosa profunda es raro, por lo que decidimos reportar el caso y hacer una revisión de la literatura.

\section{PRESENTACIÓN DEL CASO}

Paciente mujer de 52 años, agricultora, procedente de Iquitos, con antecedente de litiasis vesicular; acudió a emergencia por dos días de dolor y aumento de volumen del miembro inferior derecho, de tipo opresivo, de intensidad $8 / 10$, no irradiado, que aumentaba con el movimiento y no 
aliviaba con el reposo. Se automedica con analgésicos, sin mejoría clínica. Sin cambios en las funciones biológicas. No cuenta con hábitos nocivos.

Al examen físico, funciones vitales estables. Edema con fóvea en miembro inferior derecho, con rango articular disminuido por el dolor, signo de Homans positivo. Se palpan cinco adenopatías cervicales anteriores izquierdas no dolorosas, blandas, móviles, de aproximadamente 2 $\mathrm{cm}$ de diámetro, uno de ellos, de $4 \mathrm{~cm}$, duro, adherido a planos profundos, no doloroso (Figura 1). También se halló adenopatías inguinales bilaterales, dolorosas, blandas, no adheridas a planos profundos de $2 \mathrm{~cm}$. Palidez en piel y conjuntivas. Tórax normal. Cardiovascular normal. Abdomen, se palpa masa abdominal en epigastrio, dolorosa, dura, adherida a planos profundos de aproximadamente 7 $\mathrm{cm} \mathrm{x} 4 \mathrm{~cm}$ y borde hepático a $7 \mathrm{~cm}$ por debajo del reborde costal, doloroso a la palpación y de consistencia dura.

Ecografía Doppler del miembro inferior derecho: trombosis oclusiva en vena iliaca externa y sistema venoso fémoropoplíteo recientes.

Se realizó endoscopía digestiva alta para descartar neoplasia gástrica, la cual concluyó como pangastritis atrófica; biopsia: gastritis antro corporal crónica leve sin actividad.

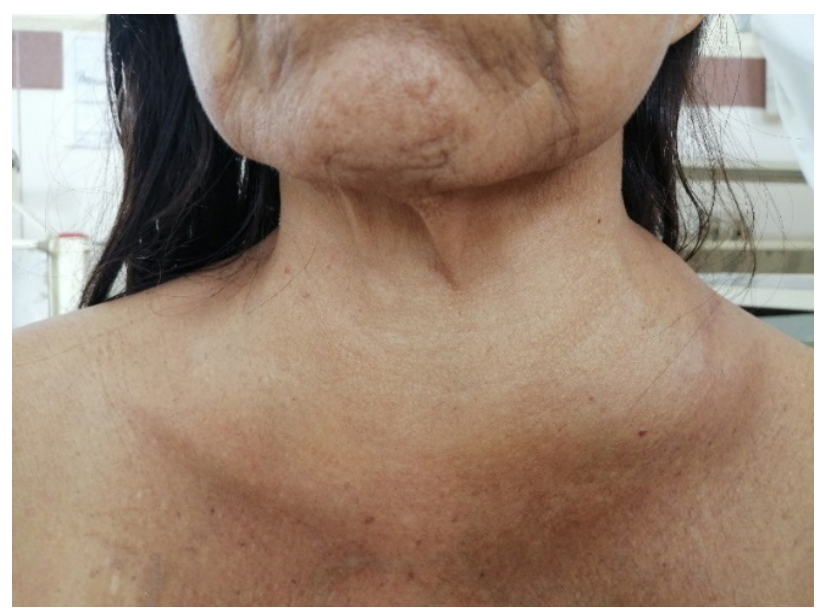

Figura. Ganglio de Virchow-Troisie.

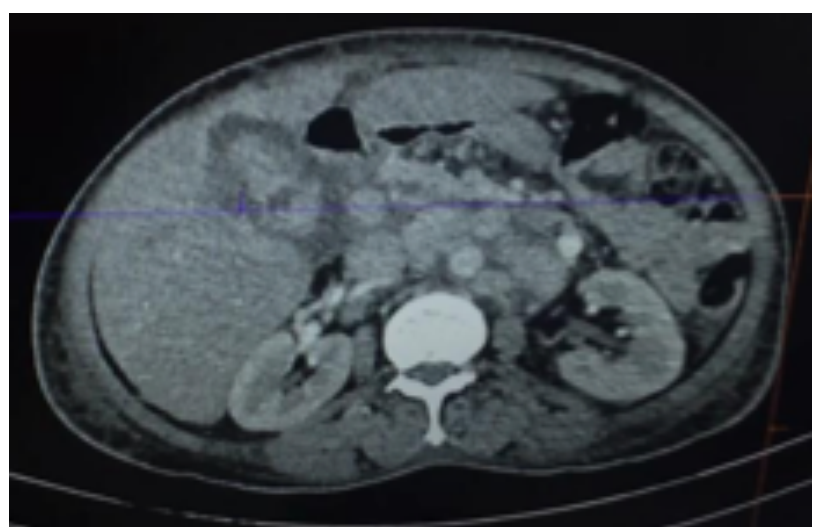

Figura 2: TEM abdominal con contraste: engrosamiento irregular de la pared vesicular.
Colonoscopía: normal. Biopsia de ganglio cervical anterior izquierdo: metástasis de adenocarcinoma.

Tomografías de tórax, abdomen y pelvis con contraste: engrosamiento irregular de la pared vesicular asociado a litiasis, a descartar proceso inflamatorio crónico vs neoformativo, adenopatías, hepatomegalia y líquido libre en cavidad abdominal (Figura 2).

Inmunohistoquímica de biopsia de ganglio: macrometástasis de carcinoma poco cohesivo con células en anillo de sello, inmunofenotipo que sugiere investigar estómago como primera posibilidad. CK-7 (+) (Figura 3).

Se decidió realizar una laparoscopía diagnóstica, teniendo como primera posibilidad de foco primario un adenocarcinoma de vesícula, correlacionando el examen físico de abdomen con los hallazgos tomográficos.

Reporte operatorio: vesícula biliar de aproximadamente $6 \mathrm{~cm} \times 4 \mathrm{~cm}$, de bordes irregulares engrosados, líquido purulento de aproximadamente 50cc en cavidad peritoneal, hígado de aspecto micronodular. Se tomó biopsia del fondo de la vesícula.

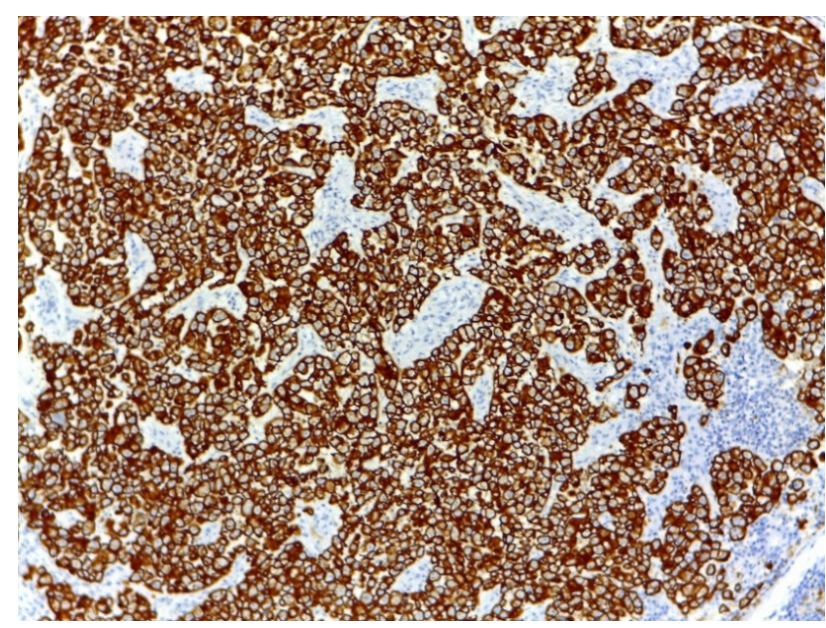

Figura 3. Inmunohistoquímica en muestra de ganglio cervical izquierdo: CK-7 (+).

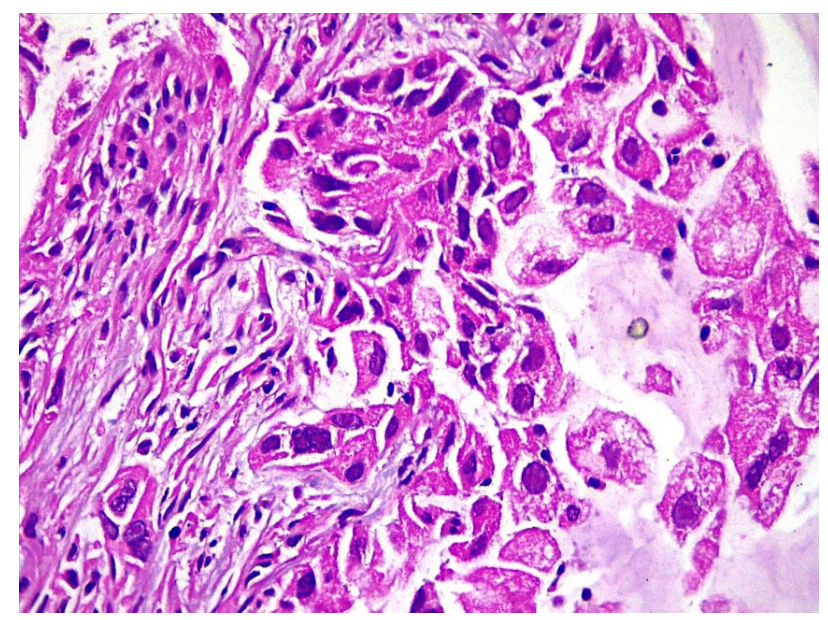

Figura 4. Muestra de vesícula biliar (HE 40X): células neoplásicas con núcleos grandes, pleomórficos e hipercromáticos, con citoplasma amplio y vacuolado; $y$, material tenuemente basofílico, de aspecto mucinoso en los espacios glandulares. 
Biopsia de vesícula: fragmentos de tejido fibroconectivo infiltrado por adenocarcinoma (Figura 4).

Se le inicio anticoagulación plena $y$, posteriormente, al encontrarse en rango terapéutico se le dio de alta con anticoagulación oral permanente y cita por consultorio externo de oncología para manejo paliativo. Un mes posterior al alta, la paciente falleció.

\section{DISCUSIÓN}

El cáncer de vesícula biliar es más frecuente en mujeres y aumenta la incidencia con la edad. Las mayores tasas de incidencia la encontramos en Chile (27/100 000), India (21,5/100 000), Pakistán (12,8/100 000) y Ecuador (12,9/100 000), siendo el área geográfica de América Latina y Asia las más asociadas con un alto promedio de incidencia de esta patología. ${ }^{5,6}$ En Perú, la incidencia ha sido reportada en 4,8 por 100000 habitantes, siendo la ciudad de Trujillo un área de alta incidencia. ${ }^{7}$

Se ha encontrado relación con múltiples factores de riesgo para desarrollar este tipo de cáncer, entre ellos tenemos: edad, sexo, colelitiasis, vesícula en porcelana, infección crónica por Salmonella, paridad alta, pólipo vesicular, obesidad y tabaquismo. ${ }^{8-10}$

Dentro de las formas más raras de diseminación se ha reportado metástasis de este cáncer a endometrio, ovario, mama, cérvix y periumbilical. ${ }^{11-15}$

El epónimo de "Ganglio de Virchow", y "Ganglio de Troisier" u otras veces llamado también "Ganglio de Virchow-Troisier", refiere al compromiso metastásico de los ganglios supraclaviculares izquierdos secundario a carcinoma avanzados, siendo el estómago como lesión primaria más frecuente. ${ }^{16}$ Pero, también lo podemos encontrar en malignidades de tórax, abdomen y pelvis ya que el lugar de aparición de esta lesión tiene relación con la desembocadura del conducto torácico en la vena subclavia izquierda, donde se drena la linfa de estas regiones a la circulación sistémica. ${ }^{17,18}$

Se ha descrito casos de Ganglio de Virchow-Troisier relacionados al cáncer de pulmón, próstata, colon, hígado, ovario, además del ya mencionado cáncer de estómago. ${ }^{19-23}$ En nuestro caso se pudo determinar mediante biopsia e inmunohistoquímica la presencia de metástasis del cáncer vesícula al ganglio en la región de la fosa supraclavicular izquierda, siendo un hallazgo poco común.

El síndrome de Trousseau es un síndrome paraneoplásico y es un evento trombótico relacionado al cáncer, que se caracteriza por flebitis superficial migratoria y trombosis venosa profunda asociada a malignidad, siendo más frecuentes los adenocarcinomas gástricos, pancreáticos y pulmonares. ${ }^{24}$ En 1865, el médico francés Armand Trousseau fue el primero en estudiar esta asociación entre eventos trombóticos y malignidad que años pasados fue solamente descrita. ${ }^{25}$

Dentro de las trombosis relacionadas al cáncer tenemos algunas formas de presentación clínica que incluyen la trombosis venosa profunda o superficial, el embolismo pulmonar, la coagulación intravascular diseminada crónica con endocarditis trombótica no bacteriana y la trombosis arterial, siendo la más frecuente el tromboembolismo venoso. Esto incluye trombosis venosa profunda y embolismo pulmonar, la presentación clínica más frecuente y se reporta del $4 \%$ a $20 \%$ en pacientes con cáncer. ${ }^{26}$

Dentro de la fisiopatología y mecanismos de producción de este síndrome de Trousseau se han descrito a las mucinas producidas por algunos adenocarcinomas. Otros mecanismos mencionados son el factor tisular; la cisteína proteinasa, que es descrita como factor procoagulante asociado al cáncer; mecanismos relacionados a hipoxia; $\mathrm{y}$, activación de oncogenes. ${ }^{27}$

En nuestro caso, la paciente ingresó por un síndrome de Trousseau y al examen físico se le encontró un ganglio de Virchow-Troisier, asociado a la edad de la paciente, ausencia de factores de riesgo para trombosis y anemia microcítica e hipocrómica se pensó como primera posibilidad una neoplasia maligna gástrica, ya que estas dos entidades se relacionan a esta patología maligna como hemos revisado en la literatura; pero, las endoscopías digestivas alta y baja fueron negativas. Para entonces ya se tenía el resultado de la patología del ganglio supraclavicular izquierdo, que concluía en metástasis de adenocarcinoma. La tomografía de abdomen con contraste nos dio luces sobre el posible foco primario ya que se notaban cambios a nivel de la vesícula biliar, por lo que se decidió estudiar dicho órgano, resultando ser el sitio primario de la neoplasia maligna y que explicaba el cuadro clínico ya descrito.

En conclusión, ante un paciente con trombosis venosa profunda y un ganglio de Virchow-Troisier debe hacernos sospechar de malignidad, siendo una opción el adenocarcinoma de vesícula biliar.

\section{REFERENCIAS BIBLIOGRÁFICAS}

1. Misra S, Chaturvedi A, Misra NC, Sharma ID. Carcinoma of the gallbladder. Lancet Oncol .2003; 4: 167-76.

2. Lai CH, Lau WY. Gallbladder Cancer. A comprehensive review. Surgeon. 2008, 6; 2: 101-110.

3. Miller G, Jarnagin WR. Gallbladder carcinoma. EJSO. 2008; 34(3):306-312.

4. Wernberg JA, Lucarelli DD. Gallbladder Cancer. Surg Clin N Am. 2014;94(2): 343-360.

5. Hickman L, Contreras C. Gallbladder cancer: diagnosis, surgical management, and adjuvant therapies. Surg Clin $N \mathrm{Am}$. 2019;99(2):337-355.

6. Schmidt MA, Marcano-Bonilla L, Roberts LR. Gallbladder cancer: epidemiology and genetic risk associations. Chin Clin Oncol. 2019;8 (4):31.

7. Salazar M, Ituarte C, Abriata M, Santoro F. Gallbladder cancer in South America: epidemiology and prevention. Chin Clin Oncol. 2019; 8(4):32.

8. Sharma A, Sharma KL, Gupta A, Yadav A, Kumar A. Gallbladder cancer epidemiology, pathogenesis and molecular genetics: Recent update. World J Gastroenterol. 2017;23(22):3978-3998. doi:10.3748/wjg.v23.i22.3978

9. Tan W, Gao M, Liu N, Zhang G, Xu T, Cui W. Body Mass Index and Risk of Gallbladder Cancer: Systematic Review and MetaAnalysis of Observational Studies. Nutrients. 2015;7(10):83218334. Published 2015 Sep 25. doi:10.3390/nu7105387 S. Kumar, S. Kumar, S. Kumar. Infection as a risk factor for gallbladder cancer. Journal of Surgical Oncology. 2006; 93:633-639.

10. Oh J, Steel M, Conklin C, et al. (July 28, 2019) Metastatic 
Gallbladder Adenocarcinoma to the Endometrium: A Case Report and Review of Literature. Cureus 11(7): e5258. doi:10.7759/ cureus. 5258

11. Sonkusare S, Vishwanath S, Kaur P, Murthi P, Krishnapriya. Occult Gallbladder Carcinoma Presenting as a Primary Ovarian Tumour: a Case Report and Review of Literature. Indian J Surg Oncol. 2018;9(4):618-621. doi:10.1007/s13193-018-0786-y

12. Kumaran D, Anamalai M, Velu U, Nambirajan A, Julka PK. Carcinoma of Gall bladder with distant metastasis to breast parenchyma. Report of a case and review of literature. J Egypt Natl Canc Inst. 2016;28(4):263-266. doi:10.1016/j.jnci.2016.06.002

13. Akhtar N, Vishnoi JR, Kumar V, et al. Adenocarcinoma of Gall Bladder Metastasis to Cervix: a Case Report with Review of Literature. J Gastrointest Cancer. 2017;48(2):190-193. doi:10.1007/ s12029-016-9815-7

14. Kim HJ, Yong HJ. Painful nodule on umbilicus: Sister Mary Joseph nodule as an unusual but important manifestation of widespread gallbladder cancer. Korean $J$ Intern Med. 2017;32(4):764-765. doi:10.3904/kjim.2016.423

15. Zdilla MJ, Aldawood AM, Plata A, Vos JA, Lambert HW. Troisier sign and Virchow node: the anatomy and pathology of pulmonary adenocarcinoma metastasis to a supraclavicular lymph node. Autops Case Rep. 2019;9(1):e2018053. Published 2019 Feb 25. doi:10.4322/ acr.2018.053.

16. Scully C, Langdon J, Evans J. Marathon of eponyms: 22 Virchow node. Oral Dis. 2012;18(1):107-108. doi:10.1111/j.16010825.2009.01554.x

17. Baumgart DC, Fischer A: Virchow's node. Lancet 2007;370: 1568. Zdilla MJ, Aldawood AM, Plata A, et al.: Troisier sign and Virchow node: the anatomy and pathology of pulmonary adenocarcinoma metastasis to a supraclavicular lymph node. Autopsy Case Rep. 2019; 9(1): e2018053.
18. Werner RA, Andree C, Javadi MS, et al. A voice from the past: rediscovering the virchow node with prostate-specific membrane antigen-targeted 18F-DCFPyL positron emission tomography imaging. Urology. 2018;117:18-21. http://dx.doi.org/10.1016/j. urology.2018.03.030. PMid:29626569.

19. Suliman MS, Singh M, Ajmeri AN, Stuart DL, Teka ST. Virchow's node: a case report of an extremely rare presentation of metastasis of adenocarcinoma with mucinous features from the colon. Int J Gen Med. 2019;12:137-140. Published 2019 Mar 29. doi:10.2147/IJGM. S201617

20. Kew MC. Virchow-Troisier's lymph node in hepatocellular carcinoma. J Clin Gastroenterol. 1991;13(2):217-219.

21. Martínez -Montero I, Domínguez-Cunchillos F, Pérez Dettoma J, et al. Ganglio de Virchow-Troisier. Diagnóstico de malignidad de tumor ovárico no filiado. Anales Sis San Navarra. 1998; 21(1):9396.

22. Stoyanov G S, Dzhenkov D L, Tzaneva M (August 30, 2019) Thrombophlebitis Migrans (Trousseau Syndrome) in Pancreatic Adenocarcinoma: An Autopsy Report. Cureus 11(8): e5528. doi: $10.7759 /$ cureus. 5528

23. Dammacco F, Vacca A, Procaccio P, Ria R, Marech I, Racanelli V. Cancer-related coagulopathy (Trousseau's syndrome): review of the literature and experience of a single center of internal medicine. Clin Exp Med. 2013;13(2):85-97. doi:10.1007/s10238013-0230-0

24. Ikushima S, Ono R, Fukuda K, Sakayori M, Awano N, Kondo K. Trousseau's syndrome: cancer-associated thrombosis. Jpn J Clin Oncol. 2016;46(3):204-208. doi:10.1093/jjco/hyv165

25. Varki A. Trousseau's syndrome: multiple definitions and multiple mechanisms. Blood. 2007;110(6):1723-1729. doi:10.1182/ blood-2006-10-053736

\section{CORRESPONDENCIA:}

Víctor A.Vargas-Rubio.

victor.vargas.rubio@upch.pe

Fecha de recepción: 23.06-2020.

Fecha de aceptación: 03-08-2020. 